\title{
Serological prevalence of Coxiella burnetii in dairy goats and ewes diagnosed with adverse pregnancy outcomes in Greece
}

\author{
George Filioussis' ${ }^{1}$, Alexandros Theodoridis², Dimitrios Papadopoulos' ${ }^{1}$, Athanasios I Gelasakis ${ }^{3}$, \\ Sotiria Vouraki ${ }^{4}$, George Bramis ${ }^{4}$, Georgios Arsenos ${ }^{4}$ \\ ${ }^{1}$ Laboratory of Microbiology \& Infectious Diseases, School of Veterinary Medicine, Faculty of Health Sciences, Aristotle \\ University, Thessaloniki, Greece \\ ${ }^{2}$ Laboratory of Animal Production Economics, School of Veterinary Medicine, Faculty of Health Sciences, Aristotle \\ University, Thessaloniki, Greece \\ ${ }^{3}$ Veterinary Research Institute of Thessaloniki, ELGO-Demeter, GR 57001, Thermi, Thessaloniki, Greece \\ ${ }^{4}$ Laboratory of Animal Husbandry, School of Veterinary Medicine, Faculty of Health Sciences, Aristotle University, \\ Thessaloniki, Greece
}

Filioussis G, Theodoridis A, Papadopoulos D, Gelasakis AI, Vouraki S, Bramis G, Arsenos G. Serological prevalence of Coxiella burnetii in dairy goats and ewes diagnosed with adverse pregnancy outcomes in Greece. Ann Agric Environ Med. 2017; 24(4): 702-705. doi: 10.26444/aaem/80706

\begin{abstract}
Introduction. Coxiella burnetii is an obligatory intracellular bacterial pathogen causing the zoonotic disease Q fever. The most common reservoirs of $C$. burnetii are wild mammals, birds and ticks. Pregnant domestic ruminants infected with this bacterium are also a major source of human infection.

Materials and method. The serological prevalence of $C$. burnetii in goats and sheep diagnosed with adverse pregnancy outcomes was assessed by undertaking a survey on 800 dairy goats and 800 dairy ewes reared in four different regions of Greece (Macedonia, Thrace, Thessaly, and Peloponnese). A stratified sampling was carried out, taking also as a criterion the age of the animals. Serum antibodies were analyzed by a commercial ELISA according to the manufacturer's recommendations. Results. Generally, there was a statistically significantly higher serological prevalence of C. burnetii (14.4\%) in goats compared to sheep (8\%). Serological prevalence was higher in adults (15.5\% in goats and $8.5 \%$ in sheep) compared to yearlings (7.4\% in goats and $4.6 \%$ in sheep). The prevalence increased significantly with age only in goats. Finally, all animals reared in Peloponnese had a prevalence significantly higher ( $21 \%$ in goats and $18 \%$ in sheep) than animals reared in the other three regions.

Conclusion. To the best of the authors' knowledge, this is the first report that associates $C$. burnetii with reproductive disturbances of domestic ruminants in Greece. However, considering the importance of coxiellosis for public health, further investigations are required on its epidemiology regarding abortion, premature delivery, stillbirth and weak offspring in small ruminants, as well as in other domestic and wild animal species.
\end{abstract}

\section{Key words}

Coxiella burnetii, reproduction, dairy sheep, dairy goats

\section{INTRODUCTION}

Coxiella burnetii (C. burnetii) is a small, short pleomorphic and obligate intracellular Gram-negative coccobacillus causing the zoonotic disease $\mathrm{Q}$ fever. The most common reservoirs of $C$. burnetii are wild mammals, birds and ticks. The dominant mode of transmission to healthy animals is through direct contact with excretions of infected animals or through tick bites [1]. In domestic ruminants, C. burnetii infection is often asymptomatic. However, infection of pregnant animals is associated with adverse pregnancy outcomes, such as abortion, premature delivery, stillbirth and weak offspring. The environment is contaminated by infected placentas at the time of parturition, leading to the persistence of the bacterium for a long time [2]. Aerosols from infected placentas, secretions or excretions of animals are a major source of transmission to humans [3]. Infected

Address for correspondence: George Filoussis, Laboratory of Microbiology \& Infectious Diseases, Schoolof Veterinarty Medicine, Faculty of Health Science, Aristotle University, 54124 Thessaloniki, Greece

E-mail: georgefilious@vet.auth.gr

Received: 29.07.2017; accepted: 21.11.2017; first published: 19.12.2017 humans develop Q fever, a zoonosis characterized by an acute form, flu-like symptoms and a chronic form with severe complications such as pneumonia, hepatitis and endocarditis [4].

Culture and molecular detection of C.burnetii are only available in reference laboratories. However, serology is considered a simple, cheap and accurate method for the diagnosis of coxiellosis in humans and animals via the detection of specific IgM and IgG antibodies [5]. The presence of $C$. burnetii in asymptomatic small ruminant populations has been known in Greece since 2008 when a survey showed a seroprevalence of $10.4 \%$ in sheep $(n=554)$ and $6.5 \%$ in goats $(\mathrm{n}=61)[6]$.

\section{OBJECTIVE}

The aim of the study was to determine the serological evidence of coxiellosis in dairy ewes and goats in Greece diagnosed with adverse pregnancy outcomes, considering the scarcity of relative data and the role that such data can play in developing preventive measures for public health. 


\section{MATERIALS AND METHOD}

The study was carried out between January 2014 - June 2015 in four different regions of Greece that host the largest national population of sheep and goat flocks. The regions were Macedonia, Thrace, Thessaly, and Peloponnese. Dairy sheep and goat farming is an important activity for these regions with socio-economic, environmental and cultural impact. A population of 200 dairy goats and 200 dairy ewes per region were included in the study. Following communication with local veterinarians in each region, the criteria for including animals in the study was clinical diagnosis of any of the following adverse pregnancy outcomes: abortion, premature delivery, stillbirth and weak offspring (known as APSW complex). All animals diagnosed with any of the latter were subjected to blood sampling within 10-15 days at the latest. Blood samples were collected from the jugular vein in designated tubes of $8 \mathrm{ml}$ (Greiner Bio-One, Kremsmunster, Austria), using a $20 \mathrm{G}$ needle (Greiner BioOne, Kremsmunster, Austria). The collected blood samples were classified into two groups: yearling animals $(<2$ years old) and adult animals ( $>2$ years old). After blood sampling, the tubes were left at ambient temperature for $30 \mathrm{~min}$ and then stored in a portable fridge (icebox). All blood samples were centrifuged at local veterinary laboratories within $6 \mathrm{~h}$ at $2,500 \mathrm{~g}$ for $15 \mathrm{~min}$; the serum samples obtained were stored at $-20^{\circ} \mathrm{C}$.

Serum samples were sent to the Laboratory of Microbiology and Infectious Diseases of the Faculty of Veterinary Medicine, Aristotle University of Thessaloniki, Greece. The serum antibody titres against $C$. burnetii were assayed using a Q fever indirect ELISA kit (ELISA Cox kit, Laboratoire Service International, Lissieu, France), according to the manufacturer's instructions. Briefly, the serum samples were incubated at room temperature for $30 \mathrm{~min}$ and then diluted in a 1:20 sample diluent. In order to detect total immunoglobulin $\mathrm{G}$ antibodies ( $\mathrm{IgG}$ ), $100 \mu \mathrm{l}$ of the diluted samples were placed on commercial ELISA plates coated with a mixture of C.burnetii phases I and II antigen. After overnight incubation at $4^{\circ} \mathrm{C}$, the plates were washed with a washing buffer. Finally, $100 \mu \mathrm{l}$ of horseradish peroxidase (HRP) conjugated antibody was added and the absorbance read using an ELISA reader at a wavelength of $450 \mathrm{~nm}$. Negative and positive controls were always included while examining serum samples. The manufacturer had previously validated the ELISA kit estimating sensitivity (Se) of 100\% and specificity (Sp) of 95\%, respectively. The criterion for considering a serum sample as positive was based on ELISA value calculated as follows: ELISA value $=100 \mathrm{x}$ (Absorbance of test sample - Absorbance value of negative control)/ (Absorbance value of positive control - Absorbance value of negative control).

Serum samples with indices $\leq 40$ were considered negative, whereas samples with indices $>40$ were considered positive. The differences of IgG between sheep and goats, age classes and regions were statistically assessed by Chi-square tests. Rogan-Gladen correction (RGC) according to the sensitivity and specificity of the ELISA test was calculated [7] using EpiTools epidemiological calculators [8]. Calculating the $95 \%$ confidence interval for each of the proportions assessed statistical uncertainty.

\section{RESULTS AND DISCUSSION}

A summary of ELISA test results on the presence of C.burnetii antibodies in animals' serum that exposed clinical signs of APSW complex is presented in Table 1. In detail, ELISA test showed in total C. burnetii seroprevalence of $14.4 \%$, CI: $12.1-17 \%$ (RGC: $9.9 \%$, CI: $7.3-12.4 \%$ ) in goats and $8 \%$, CI: 6.3-10.1\% (RGC: $3.2 \%$, CI: $1.2-5.1 \%$ ) in sheep. Similar studies dealing with epidemic reproductive disorders have indicated that C. burnetii seroprevalence ranges between $31-93 \%$ in goat flocks and 9-25\% in sheep flocks [9]. Furthermore, in the presented study, statistically significant differences were found between goat and sheep seroprevalences $\left(\chi^{2}=16.36\right.$, $\mathrm{p}$-value $=0.000 \leq 0.05$ ). The latter result can be attributed to the intrinsic susceptibility of goats to coxiellosis that is clinically manifested with abortion and/or reproductive problems [10].

ELISA seroprevalence-age associated patterns differed between the two ruminant species tested in the present study (Tab. 1). Seroprevalence of C. burnetii appeared to be higher in adults goats (15.5\%, CI: $13-18.3 \%$, RGC: $11 \%$, CI: $8.2-13.8 \%$ ) than in yearlings (7.4\%, CI: $3.8-13.9 \%$, RGC: $2.5 \%$, CI: $-2.7-7.7 \%)$, as well as in adults sheep (8.5\%, CI: 6.7$10.8 \%$, RGC: $3.7 \%$, CI: $1.5-5.9 \%$ ) than in yearlings (4.6\%, CI: $2-10.4 \%$, RGC: $<0.0)$. Differences, however, were statistically significant only for goats $\left(\chi^{2}=16.36\right.$, $p$-value $\left.=0.001 \leq 0.05\right)$. Similar age-related C. burnetii serological patterns in sheep and goats that exposed clinical signs of APSW complex have been reported previously [11]. Such results indicate that females used as replacements (less than 1 year old) are probably less exposed to the pathogen until the lambing period when the agent shows higher exposure rates due to increased excretion [12]. Moreover, as a consequence of a higher probability of contact, pathogen contact rate increases with age, a feature observed mainly in goats.

The region of herd origin was also indicated as a significant risk factor for seropositivity (Tab. 1). The lowest seroprevalence was observed in goats (11\%, CI: 7.4-16.1\%, RGC: $6.3 \%$, CI: $1.8-10.9 \%$ ) and sheep (3.5\%, CI: $1.7-7 \%$, RGC: $<0.0)$, reared in Macedonia. Additionally, the highest seroprevalence was observed in goats (21\%, CI: $15.9-21.2 \%$, RGC: $16.8 \%$, CI: $10.9-22.8 \%)$ and sheep (18\%, CI: $13.9-23.9 \%$, RGC: $13.7 \%$, CI: 8.1-19.3\%) reared in Peloponnese.

Finally, significant differences were found between regional seroprevalence, both in sheep $\left(\chi^{2}=36.82\right.$, $p$-value $=0.000 \leq 0.05)$ and goats $\left(\chi^{2}=10.02\right.$, $p$-value $\left.=0.018 \leq 0.05\right)$. The latter was expected since the Greek sheep farming sector is characterized by a diversity of production systems, ranging from extensive, pastoral and semi-extensive to intensive. Specifically, the extensive production systems that are situated mainly in mountainous areas, influence the transmission of C. burnetii in small ruminants through wildlife/livestock contact or through arthropod vectors.

In the presented study, ELISA was used as a diagnostic tool because compared to other serological techniques its reliability is not limited to the high variability of C. burnetii excretion by animals throughout the year. During recent decades, different serological techniques, such as indirect fluorescence assay (IFA), complement fixation tests (CFT) and ELISA, have been employed worldwide to detect C. burnetii antibodies. In most cases, contact of this pathogen at the population level was screened by a highly sensitive test; positive samples were confirmed by using a highly special tool. In contrast, there has been no need for any 


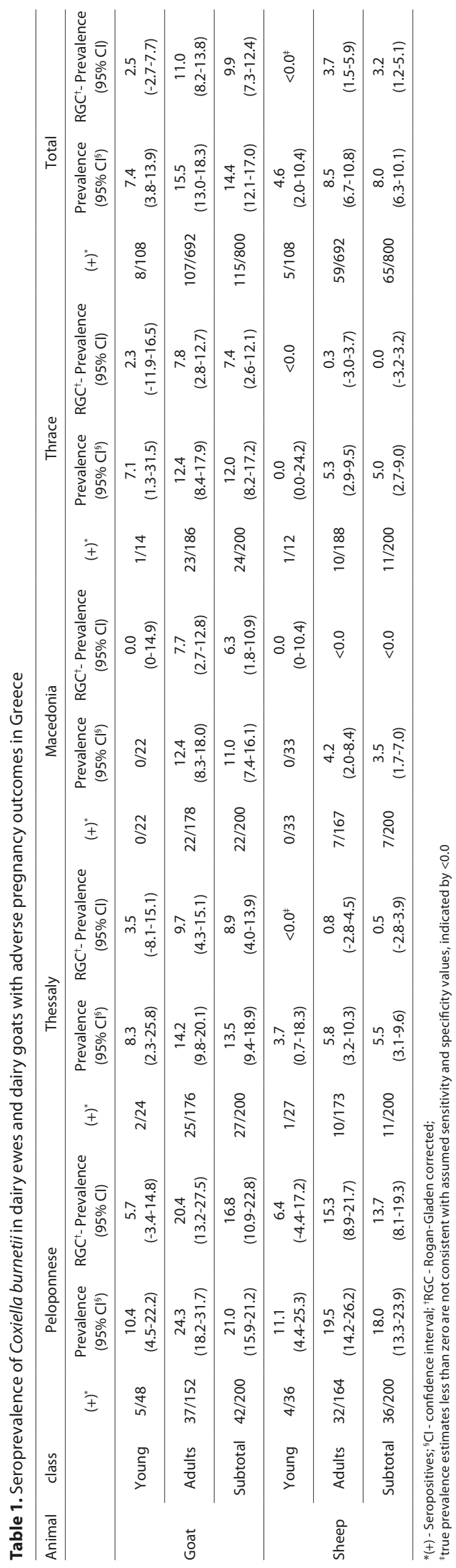


further confirmation of the results in this study since the validated commercial ELISA kit used had high sensitivity and specificity.

\section{CONCLUSION}

To the best of the authors' knowledge, this is the first report that associates $C$. burnetii with reproductive disturbances of domestic ruminants in Greece. Considering the importance of coxiellosis for public health, further investigations are required on its epidemiology regarding APSW syndrome in small ruminants, as well as in other domestic and wild animal species. Such studies should focus on the isolation of the pathogen from animals with adverse pregnancy outcomes, as well as genomic evaluation of the isolates. As $\mathrm{Q}$ fever can be a fatal disease, professionals working in the health system need to regularly monitor and report the occurrence of the pathogen. In particular, the results of the presented study suggest that domestic ruminants with reproductive disturbances should be checked for the presence of C. burnetii.

\section{Acknowledgements}

The study was partially funded by CEVA HELLAS LLC.

\section{REFERENCES}

1. Aitken ID, Bögel K, Cracea E, Edlinger E, Houwers D, Krauss H, et al. Q fever in Europe: current aspects of aetiology, epidemiology, human infection, diagnosis and therapy. Infection. 1987; 15: 323-327.

2. Baud D1, Greub G. Intracellular bacteria and adverse pregnancy outcomes. Clin Microbiol Infect. 2011; 17: 1312-1322.

3. Angelakis E, Raoult D. Q fever. Vet Microbiol. 2010; 140: 297-309.

4. Parker NR, Barralet JH, Bell AM. Q fever. Lancet. 2006; 367: 679-688.

5. Fournier P-E, Raoult D. Comparison of PCR and serology assays for early diagnosis of acute Q fever. J Clin Microbiol. 2003; 41: 5094-5098.

6. Pape M, Bouzalas EG, Koptopoulos GS, Mandraveli K, ArvanitidouVagiona M, Nikolaidis P, et al. The serological prevalence of Coxiella burnetii antibodies in sheep and goats in northern Greece. Clin Microbiol. 2009; 15: 146-147.

7. Rogan WJ, Gladen B. Estimating prevalence from the results of a screening test. Am J Epidemiol. 1978; 107: 71-76.

8. Sergeant ESG n. Epi Tools - Sample size to estimate a proportion with specified precision. Available from: http://www.epitools.eu/content. php?page $=1$ Proportion

9. Ruiz-Fons F, Astobiza I, Barandika JF, Hurtado A, Atxaerandio R, Juste RA, et al. Seroepidemiological study of $Q$ fever in domestic ruminants in semi-extensive grazing systems. BMC Vet Res. 2010; 6: 3.

10. Valergakis GE, Russell C, Grogono-Thomas R, Bradley AJ, Eisler MC. Coxiella burnetii in bulk tank milk of dairy cattle in south-west England. Vet Rec. 2012; 171: 1-2.

11. García-Pérez AL, Astobiza I, Barandika JF, Atxaerandio R, Hurtado A, Juste RA. Investigation of Coxiella burnetii occurrence in dairy sheep flocks by bulk-tank milk analysis and antibody level determination. J Dairy. 2009; 92: 1581-1584.

12. Maurin M, Birtles R, Raoult D. Current knowledge of Bartonella species. Eur J Clin Microbiol Infect Dis. 1997; 16: 487-50. 